\title{
Web-based Coordination for E-Commerce
}

\author{
XiguoZhang, GuiheWang, LiminFan \\ Department of Mechanical Engineering \& Automation, Eastern LiaoNing \\ University \\ Dandong, 118000 , P.R.China \\ guihewang@163.com
}

\begin{abstract}
As the e-commerce environment becomes more pervasive and dynamic, coordination among companies are required more frequently than ever. We give the definition of coordination as well as the model of ecommerce based on web; the communication in e-commerce is described; the function of coordination, include the architecture, execution and control of coordination is discussed. At the same time, we analyze the business process perspective and develop a coordination system based web for e-commerce, based which, the process of problem solving is analyzed.
\end{abstract}

\section{Introduction}

The advent of e-ecommerce has revolutionized the way that contemporary business operates [1]. With the development of the Internet, e-commerce has become more important. Dynamic e-commerce is concerned with the integrate systems across intranets, extranets, and the Internet in a dynamic fashion[2]. Equipped with advanced Internet technologies and standards, participants in dynamic e-business are able to externalize a company's business processes in a standard way and utilize business processes provided by other parties to create new applications or business flows by integrating such internal and external processes dynamically[3]. The use of Internet services lead to considerable savings time and cost for construction projects. Although, web services technology have provided a flexible deployment approach to facilitate $\mathrm{B} 2 \mathrm{~B}$ and $\mathrm{B} 2 \mathrm{C}$ interactions[5], coordination is need frequently than ever among companies as the business environment becomes more dynamic. The required amount of information processing and communication in those cases can easily exceed a single human being's capability, especially when there are many negotiation issues and partners [6]. Therefore, systems supporting and automating business negotiations have a great potential value.

Please use the following format when citing this chapter:

7hang, X., Wang, G., Fan, I., 2007, in IFIP International Federation for Information Processing, Volume 251, Integration and Innovation Orient to L-Society Volumel, Wang, W. (Lds), (Boston: Springer), pp. 515-522. 


\section{Web services for e-commerce}

\subsection{The model of e-commerce}

E-commerce application can interact with applications of external trading partners or other internal cross-functional applications by exchanging messages [7]. Figure 1 illustrates the usage of Web services in e-commerce.

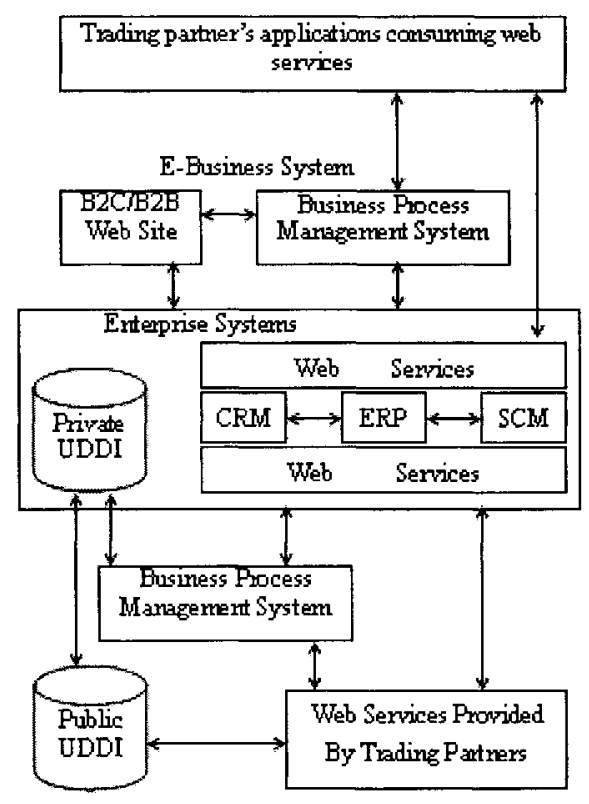

Fig.l. The e-commerce of web-based

A business process can expose its functionality as Web services and consume the functionality of other processes. Web services play an important role in enhancing business-to-consumer (B2C) and business-to-business (B2B) integration by allowing trading partners to gain access to data or business functions in the legacy systems and enterprise software systems either via Web services wrappers of these systems directly or via Business Process Management System (BPMS). An enterprise can provide Web services to its trading partners for delivering real-time data or embed its business services directly as part of partners' services to their customers. Activities in business processes can also be implemented as Web services. The BPMS uses private and public UDDI service registries to store, search, and select business partners' Web services for business process integration. Web services are used to support dynamic e-business consisting of dynamically configured business networks. 


\subsection{E-commerce communication}

Web services are self-contained and modular applications that can be described, published, located and invoked over the web. Web services perform encapsulated business functions ranging from simple request-reply to full business process interactions, which can reduce human interaction through the embedded capability of services discovery and binding, which are standards-based and suited to build common infrastructure to reduce the barriers of business integration. The dynamic nature of Web services also opens up new opportunities for businesses, which can application in following areas:

1) Between enterprises: Web services can provide services via a standard interface to customers and business partners. In addition to exchanging plain transaction data, business process level integration with trading partners will become a real possibility.

2) Within an enterprise: Web services can accelerate the speed and reduce the cost of integration with various internal applications and systems. They also have the potential of reducing programming skill requirements and improving service asset reuse thanks to its service-oriented structure based on open standards.

3) Between an enterprise and its (internal or external)end-users: Web services can deliver a better user experience, integrate diverse content and reduce the cost of content variety to a variety of user devices due, in part, to the availability of Web services-enabled applications, data sources and devices.

\subsection{Coordination definition}

The first step of the system-supported coordination is to establish shared understanding of the rules of the coordination. Thus, the coordination process must be clearly defined and easily interpreted. However, defining coordination processes from scratch takes time and efforts and checking the correctness of the defined process requires expertise. The marketplace is in the best position to define coordination processes because it can accumulate know-how in designing coordination processes while supporting various transactions. The coordination processes defined and given by the marketplace have also a clear advantage in accessibility and reusability. The opponent's utility function is assumed of the following product form:

$$
u=\prod_{i} f_{i}^{w_{i}}
$$

Where $f_{i}$ and $\mathrm{w}_{i}$ corresponds to the utility value and weight of issue $i$. Note that this objective function differs from the linear combination formulation of the multiattribute utility theory that most existing coordination system employ. Opponent's feedbacks are used to adjust the weights in order to reveal its underlying preferences. This can be achieved by comparing the changes of consecutive offers, namely:

$$
R_{i}=\left|\frac{f_{i}(t)-f_{i}(t-1)}{f_{i}^{\prime}-f_{i}^{\prime}(t-1)}\right|,
$$

Where $f_{i}(\mathrm{t})$ and $f^{\prime},(\mathrm{t})$ denotes respectively the user's and the opponent's proposed values for issue $i$ at round $t$. Note that this ratio reveals the opponent's relative preference compared with the user upon issue $i$. A large ratio suggests that issue $i$ is 
more important to the opponent than the user in a quantitative measure. Table 1 shows the weight range of categories.

Once the coordination partners agree on the coordination, an instance of the coordination process needs to be instantiated, controlled, and monitored. The coordination process can be executed and controlled by invoking and scheduling activities according to the process definition. It can also enforce the commitment of coordination partners by providing a non-repudiation mechanism. The harmonization is also the best way to execute and control coordination processes because it can provide a both-win perspective.

Table 1. The Weight Value of Categories

\begin{tabular}{cc}
\hline Categories & Weight range \\
Important & $0.75-0.95$ \\
Less & $0.35-0.70$ \\
important & \\
No important & $0.10-0.30$ \\
\hline
\end{tabular}

A business coordination process is often ad hoc in nature because the relationships between negotiation partners are dynamic and the most appropriate coordination process is dependent on the specific case. The proposed Web Servicesenabled marketplace architecture is a nice solution to understandability and information sharing issues and intentionally leaves internal decision and collaboration processes to the coordination partner side. However, the generation of new coordination processes and their modification are not well supported. It is often the case that at least minor changes to the given coordination process are desirable in order to accommodate company- or case specific issues. The coordination through general communication channels provides the maximum flexibility in process but the minimum codified information on the process and exchanged data. The coordination processes are in the following way:

1) The buyers and suppliers find coordination partners.

2) Partners make an agreement on the coordination process. Say a set of process $P=\left\{P_{I}, \ldots, P_{n}\right\}$ and a set of attributes $R=\left\{R_{l}, \ldots, R_{m}\right\}$. The attribute $R_{j}$ contains patterns $a_{k}$ (i.e. $\left.R_{j}=\left\{a_{l}, \ldots, a_{p}\right\}\right)$.Each process $P_{i}$ is a combination of the patters for the attributes (i.e. $P_{i}=\left\{a_{l}, \ldots, a_{m}\right\}$ where $a_{j} \in R j$ ).

3) The partners start with a coordination process which may be either one of the given coordination processes $\mathrm{P}_{\mathrm{i}}$ or a new coordination process $p_{k}$ created by the process composition tool.

4) Coordination partners perform the coordination until reaching an agreement or breaking off the coordination. Using the propriety tool, users can easily generate a new process $p_{k}$ by selecting patterns for coordination process attributes $R=\left\{R_{l}, \ldots, R_{m}\right\}$, modifying the attributes in the ready-made process. 


\section{The system of e-commerce}

\subsection{The architecture of the system}

The distributed prototype system uses a J2EE based multi-layer architecture. Figure2 depicts its architecture, the prototype system consists of four tiers implemented on the J2EE platform for delivery of online information.

1) Client layer: The thin Web clients are used in the architecture. They are the Web browsers to assemble checking requests with the target server URL (Uniform Resource Locator) and send them to the server through the HTTP protocol. The checking results in dynamic HTML are also rendered at the client tier.

2) Web layer: It consists of a Web container to host all the Web components mentioned. The presentation logic of the prototype is processed at the Web layer, including the delivery of the dynamic Web content to client. Both Servlet and JSP technologies are implemented in the prototype together with Java bean.

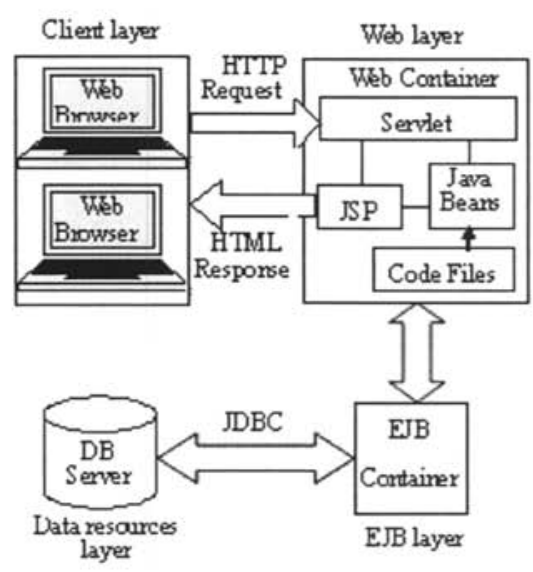

Fig.2. The architecture of e-commerce system

3) EJB layer: Enterprise Java beans are developed at this layer for responding to user requests from the Web layer through use of building rules in the knowledge base at the data resources layer. All application logic for building design support services is implemented at this layer.

4) Data resources layer: It includes a database server to hold and maintain the content of the knowledge. JDBC (Java Database Connection) is used for the connection between the EJB layer and the database server. JDBC also provides support for distributed transaction management and database access security for the prototype. 


\subsection{Coordination model}

The traditional contract net is employed to handle coordination, which includes the inviting, bidding and awarding stages. Figure 3 shows a collaborative coordination model based on coordination and bidding. Comparing with the traditional systems, this model enables multi-contract coordinate of to improve the overall performance, resulting in a batch of contracts that are consistent and coherent to the ultimate goal--fulfilling customer orders. As shown in figure 3, a customer order is first mapped to configuration unit.

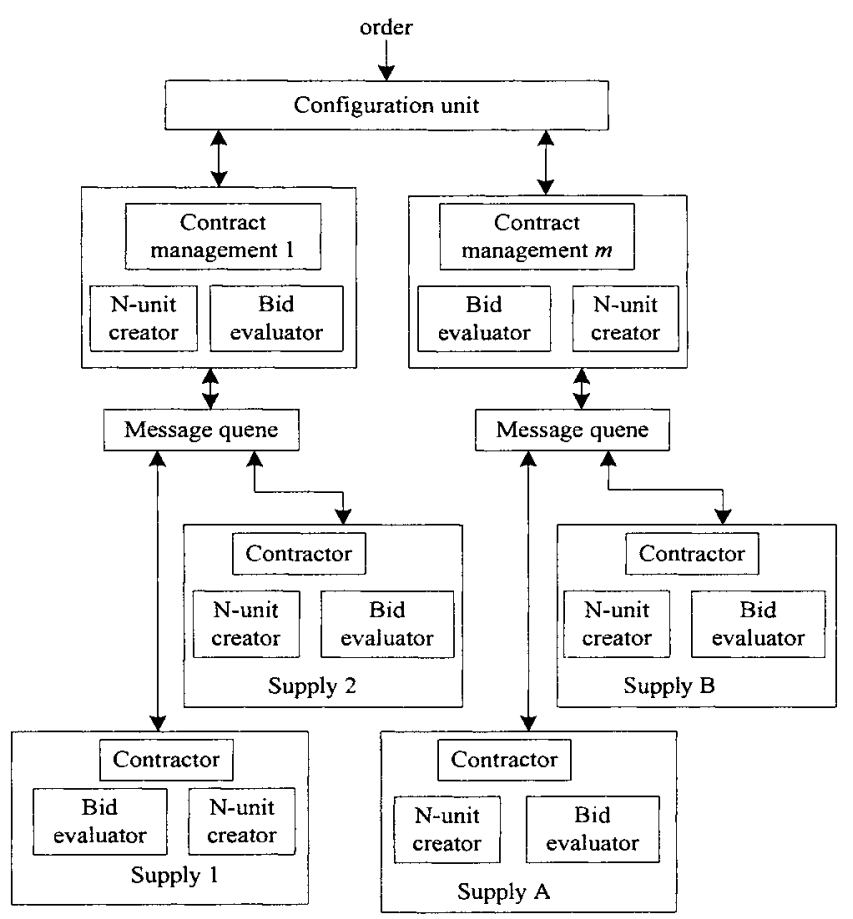

Fig.3. The coordination model

Then the system starts to find proper trading partners for individual contract Manager. Each contract unit would bid for the relevant task based on its production capability and resource availability. A separate configuration unit is used to solve the possible conflicts among individual tasks. The system employs four types of functional units: configuration unit, contract manager, information server, and coordination unit. The configuration unit is responsible for coordinating the product fulfillment process.

The contract manager is in charge of specific evaluators and manages the coordination process to reach a contract for this evaluator. The information server 
assists the contract manager in inviting the candidate suppliers and provides a message routing service. The coordination unit executes a certain coordination process with a supplier.

\subsection{Problem Solving Process}

The blackboard provides a shared data structure for knowledge sources to post solution components (e.g. new production schedules, new capacity allocations, new parties names, etc.), analysis results (e.g. resource/capacity utilization, failure records, etc.), and coordination/communication status information. It is partitioned into an arbitrary number of contexts, which corresponds to different sets of working assumptions (e.g. sets of orders need to be scheduled, available resource capacities, etc.) and different solutions. Within each context, a summary of the current state of the solution is maintained in the form of a set of "unresolved issues". An unresolved issue is an indication that a particular aspect of the current context solution is incomplete, inconsistent or unsatisfactory. For example, a request for bid still needs to be evaluated, or a promised delivery date is violated. Problem solved process is shown in figure4.

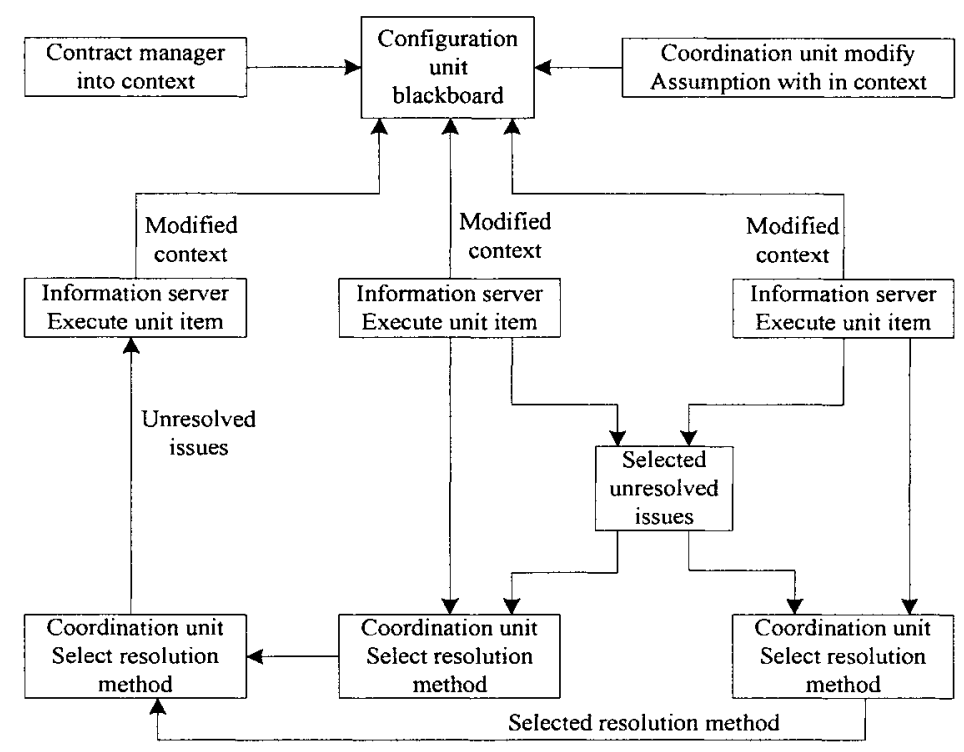

Fig.4. Flow of problem solving

All problem-solving activities within the system architecture are triggered by either the incorporation of a new event or modification of an assumption, both of which can be performed by either the end user or the unit. The module-activation service is invoked whenever there are problem-solving tasks on the agenda remaining to be executed, or initiated by units within the system. Knowledge sources 
serve as the primary problem solvers in the system. They communicate the real-time results by posting new information to the blackboard and modifying existing information. Each domain-level knowledge source acts primarily as a server that supports a variety of problem-solving services.

\section{Conclusions}

Internet and new software development technologies created new opportunities for the design and deployment of systems capable of supporting coordinators. As the business environment becomes more dynamic, coordination amount companies are required more frequently than ever, systems supporting and automating business coordination have a great potential value. The biggest advantage of the Web Services-enabled coordination is the increased size of the pool of potential partners. Through the Web Services interfaces, heterogeneous systems of trading partners can dynamically link with each other. Considering the degree of automation achieved in other business process domains, the importance of managing coordination to achieve more efficient business operations has become even higher.

\section{Acknowledgement}

The authors thank the Chairman of the Editorial Board and anonymous referees for their helpful comments on the earlier version of this work paper. This work was supported by the key Science and Technology Project from Liaoning Province under the grand No.: 2003220025.

\section{References}

1. G. Francisco, V. Rafael, and M. Rodrigo, An integrated approach for developing eommerce applications, Expert Systems with Applications 2812 (2000) 223-235

2. L. Yu, L. Liu, and X. Li, A hybrid collaborative filtering method for multiple-interests and multiple-content recommendation in e-commerce, Expert Systems with Applications 2616 (2005) 67-77

3. A. Basu, and S. Muylle, Online support for commerce processes by Web retailers, Decision Support Systems 344 (2003) 379- 395

4. A. Kambil, A. Kalis, M. Koufaris, and H.C. Lucas, Influences on the corporate adoption of Web technology, Communications of the ACM 4311 (2000) 264- 271

5. H. Kreger, Fulfilling the Web services promise, Communications of the ACM 466 (2003) 29-34

6. R.D. Van der Mei, R. Hariharan, W. Ehrlich, and P.K. Reeser, Performance of Web servers in a distributed computing environment, Engineering in the Internet 812 (2001) 125-134

7. E. Cohen, H. Kaplan, and J.D. Oldham, Managing TCP connections under persistent HTTP, Computer Networks 3122 (1999) 1709-1723

8. Y. Huang and J.Y. Chung, A Web services-based framework for business integration solutions, Electron Commerce Research 3418 (2003) 15-26 